Louisiana State University

LSU Digital Commons

6-30-2006

\title{
The Crystal Structure of the Transcriptional Regulator HucR from Deinococcus radiodurans Reveals a Repressor Preconfigured for DNA Binding
}

\author{
Tee Bordelon \\ Louisiana State University \\ Steven P. Wilkinson \\ Louisiana State University \\ Anne Grove \\ Louisiana State University \\ Marcia E. Newcomer \\ Louisiana State University
}

Follow this and additional works at: https://digitalcommons.Isu.edu/biosci_pubs

\section{Recommended Citation}

Bordelon, T., Wilkinson, S., Grove, A., \& Newcomer, M. (2006). The Crystal Structure of the Transcriptional Regulator HucR from Deinococcus radiodurans Reveals a Repressor Preconfigured for DNA Binding. Journal of Molecular Biology, 360 (1), 168-177. https://doi.org/10.1016/j.jmb.2006.05.005 


\title{
The Crystal Structure of the Transcriptional Regulator HucR from Deinococcus radiodurans Reveals a Repressor Preconfigured for DNA Binding
}

\author{
Tee Bordelon, Steven P. Wilkinson, Anne Grove \\ and Marcia E. Newcomer*
}

Department of Biological Sciences, Louisiana State University, Baton Rouge LA 70803, USA

\begin{abstract}
We report here the $2.3 \AA$ resolution structure of the hypothetical uricase regulator (HucR) from Deinococcus radiodurans R1. HucR, a member of the MarR family of DNA-binding proteins, was previously shown to repress its own expression as well as that of a uricase, a repression that is alleviated both in vivo and in vitro upon binding uric acid, the substrate for uricase. As uric acid is a potent scavenger of reactive oxygen species, and as $D$. radiodurans is known for its remarkable resistance to DNA-damaging agents, these observations indicate a novel oxidative stress response mechanism. The crystal structure of HucR in the absence of ligand or DNA reveals a dimer in which the DNA recognition helices are preconfigured for DNA binding. This configuration of DNA-binding domains is achieved through an apparently stable dimer interface that, in contrast to what is observed in other MarR homologs for which structures have been determined, shows little conformational heterogeneity in the absence of ligand. An additional amino-terminal segment, absent from other MarR homologs, appears to brace the principal helix of the dimerization interface. However, although HucR is preconfigured for DNA binding, the presence of a stacked pair of symmetry-related histidine residues at a central pivot point in the dimer interface suggests a mechanism for a conformational change to attenuate DNA binding.
\end{abstract}

(C) 2006 Elsevier Ltd. All rights reserved.

Keywords: DNA-binding protein; protein structure; MarR; repressor; X-ray crystallography
D. radiodurans is a non-pathogenic, Gram positive, non-motile, aerobic mesophile best known for its remarkable ability to withstand both acute and chronic exposure to high levels of ionizing radiation that often leads to double-strand DNA breaks. D. radiodurans is also highly resistant to other sources of DNA damage, including ultraviolet radiation, desiccation, and oxidative stress. ${ }^{1}$ $D$. radiodurans was found to encode orthologs to almost all known bacterial genes involved in stress responses, including $\mathrm{pH}$, desiccation, temperature, phage, toxin, antibiotic, and oxidative stresses. 1,2

The $D$. radiodurans $\mathrm{R} 1$ gene designated hucR (hypothetical uricase regulator) encodes a 181 amino acid residue protein that has been suggested to play a critical role in the cellular response to oxidative stress. ${ }^{3,4}$ The unique binding site for HucR overlaps the promoters of both $h u c R$ and a divergently oriented gene encoding a uricase, suggesting 
repression of both genes by HucR. Indeed, in vivo analyses showed that repression of both $h u c R$ and uricase genes is relieved in the presence of uric acid, and in vitro studies showed attenuated HucR-DNA complex formation upon addition of uric acid $\left(K_{\mathrm{d}}\right.$ $\sim 12 \mu \mathrm{M})$. Uric acid, an intermediate in purine metabolism, has been shown to serve as a scavenger of reactive oxygen species (ROS), with the reaction of uric acid with ROS resulting in the production of allantoin, which is also the product of uricasemediated degradation of uric acid. ${ }^{5-7}$ Notably, the limited solubility of uric acid demands that its concentration be tightly regulated.

HucR belongs to the multiple antibiotic resistance regulator (MarR) family of transcriptional regulators and shares $29 \%$ sequence identity with the prototypical member MarR from Escherichia coli, 34\% identity with Pseudomonas aeruginosa MexR, and less than 20\% with Bacillus subtilis OhrR, for which structures have been reported. ${ }^{8-10}$ MarR homologs are homodimers that bind sequence-specific palindromic or pseudopalindromic DNA via a wingedhelix motif. Most MarR family members are transcriptional repressors; however, some (e.g. BadR and ExpG) have been shown to activate transcription. ${ }^{11,12}$

The MarR family is a particularly significant class of transcriptional regulators, as the genes they regulate often confer increased resistance to multiple antibiotics (hence the name), organic solvents, household disinfectants, detergents, and oxidative stress agents. ${ }^{13}$ For example, in the absence of the appropriate stimulus, E. coli MarR negatively regulates the marRAB operon and repression of this operon is alleviated by exposure to a variety of phenolic compounds, most notably sodium salicylate. ${ }^{14}$ Similarly, MexR negatively regulates an operon in $P$. aeruginosa that, when expressed, encodes for a tri-partite multi-drug efflux system that results in an increased resistance to multiple antibiotics, including tetracycline, $\beta$-lactams, chloramphenicol, novobiocin, trimethoprim, sulfonamides, and fluoroquinolones. ${ }^{15,16}$ Similar to the proposed cellular function of HucR, the MarR homolog OhrR mediates a response to oxidative stress; however, for OhrR, it is oxidation of a lone cysteine residue by organic hydroperoxides that abrogates DNA binding. ${ }^{17,18}$

The structures of MarR(1JGS), ${ }^{8} \operatorname{MexR}(1 \mathrm{LNW}),{ }^{9}$ SlyA-like protein (1LJ9), ${ }^{19}$ and OhrR(1Z9C) ${ }^{10}$ have revealed a common tweezers-like dimeric structure in which the winged-helix domains are at the tips. The dimerization domain, the hinge of the tweezers, is formed by the amino and carboxyl terminal helices, and the ligand-binding site, according to the MarR structure, is fully integrated into the DNAbinding domain. Although MarR homologs share significant structural homology, the mechanism by which they bind their cognate DNA sequences differs, as demonstrated by DNA footprint analyses in which members such as MexR and HucR bind cognate sites located on the same face of the DNA, while MarR binds sites located on opposite faces of the DNA double helix. ${ }^{3,20,21}$ Furthermore, structural studies of MexR and OhrR have suggested that flexibility at the dimer interface is a hallmark of these proteins as (1) the crystal structure of MexR in the absence of ligand revealed four dimers that differ significantly in conformation at the dimer interface, ${ }^{9}$ and (2) the structures of reduced OhrR in the absence and in the presence of DNA reveal a significant reorientation of an $\alpha$-helix at the dimerization interface. ${ }^{10}$

The sequence of HucR defines it as a member of a subgroup of the MarR family, as HucR has an additional 35 amino acid residues at the amino terminus. None of the MarR homologs in the Protein Data Bank includes this extension. Furthermore, there are distinct functional differences between HucR and the MarR homologs for which structures have been determined. While HucR recognizes a single DNA sequence with high affinity (HucR binds its cognate DNA with subnanomolar affinity), MarR binds two homologous DNA sequences, and OhrR binds its cognate DNA with more than tenfold lower affinity compared to HucR. In an effort to define elements of structure that confer functional differences in MarR homologs further, we determined the crystal structure of HucR to $2.3 \AA$ resolution by multi-wavelength anomalous diffraction (MAD). In contrast to what is observed for OhrR in the absence of DNA, the dimer interface of HucR, which is braced by a helix from the HucRspecific additional amino-terminal segment, confers a disposition of DNA-binding domains that is fully compatible with DNA binding. In addition, a stacking interaction of symmetry-related histidine residues at the dimer interface suggests that DNA binding can be attenuated by $\mathrm{pH}$ changes, consistent with titration of histidine. Significantly attenuated HucR-DNA interactions at $\mathrm{pH} 5.0$ support a functional role of the dimer interface in modulating DNA binding.

\section{Results and Discussion}

\section{Overall structure of HucR}

The crystal structure of the HucR dimer was determined to $2.3 \AA$ and refined to a final $R_{\text {cryst }}$ of $23.5 \%$ and $R_{\text {free }}$ of $29.0 \%$. HucR has a "saddle-like" shape with overall dimensions of approximately $60 \AA$ wide, $40 \AA$ tall and $40 \AA$ deep with respect to the orientation shown in Figure 1(a). An additional $\alpha-$ helix in HucR is positioned between the dimerization and DNA-binding domains such that the canonical tweezers-like form of the superfamily has a broadened and rounder appearance. The protein is largely $\alpha$-helical (55\% $\alpha$-helix, 5\% $\beta$-strand) with the topology $\alpha 1-\alpha 2-\alpha 3-\beta 1-\alpha 4-\alpha 5-\beta 2-\beta 3-\alpha 6-\alpha 7$. The first helix, $\alpha 1$, has no counterpart in any other MarR family member for which structures have been described, and appears to brace the amino-terminal end of $\alpha 2$, which connects the dimerization and 
(a)

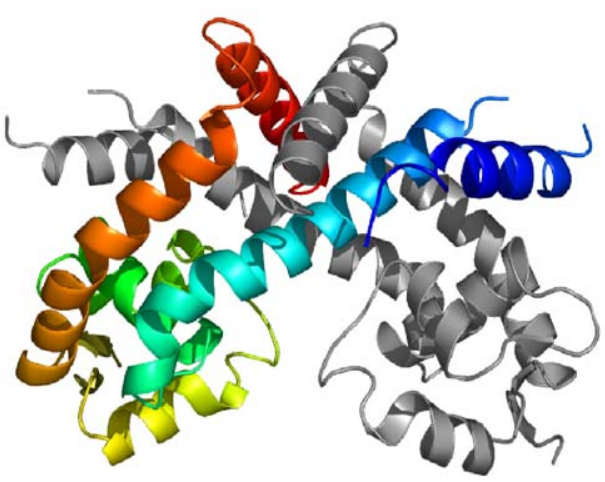

(b)

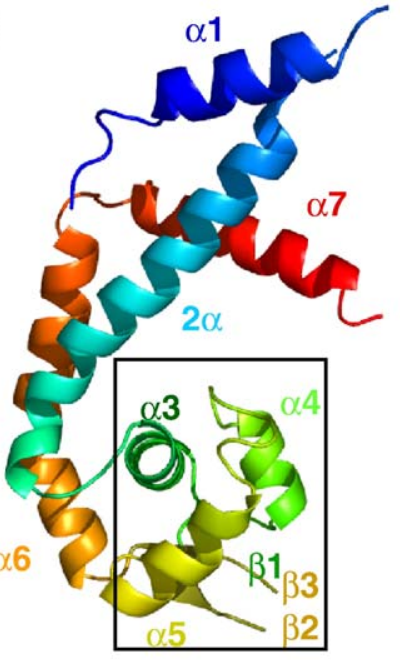

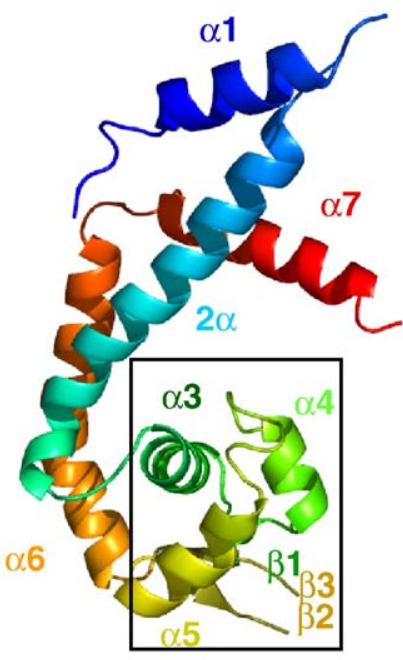

(c)

Potential

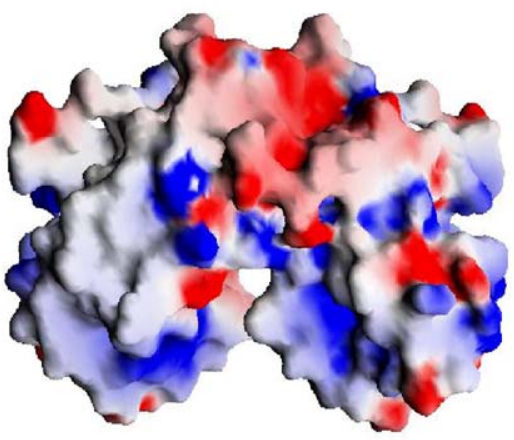

Figure 1. The strucure of of HucR. (a) A cartoon drawing of the HucR dimer with one monomer colored according to sequence number (blue $\rightarrow$ red, $N \rightarrow C$ ) and its mate in gray. (b) A stereo view of the monomer with the elements of secondary structure labeled. The DNA-binding domain is enclosed by the box. (c) The corresponding electrostatic surface representation (red, negative; white, neutral; blue, positive) for the dimer view in (a). The histidine residues are not protonated for the calculation.

DNA-binding domains. Helices from the amino and carboxyl termini ( $\alpha 2, \alpha 6$, and $\alpha 7)$ form the dimerization domain, while the DNA-binding domain is composed of $\alpha 3, \beta 1, \alpha 4, \alpha 5, \beta 2$, and $\beta 3$ (Figure 1(a) and (b)). As predicted, the structure reveals that HucR adopts a winged-helix fold similar to that seen in the structures for MarR, MexR, and OhrR. ${ }^{8-10}$ Consistent with that of other winged-helix proteins, $^{22}$ the electrostatic surface potentials of HucR surrounding the recognition helix $(\alpha 5)$ and the wing regions are electropositive, and are likely to interact with the negatively charged DNA (Figure 1(c)).

\section{The DNA-binding domain}

The DNA-binding domain (residues 65-133) corresponds to a contiguous stretch of polypeptide flanked by regions that form the dimerization domain and is comprised of $\alpha 3, \beta 1, \alpha 4, \alpha 5, \beta 2$, and $\beta 3$ (Figure 1). The winged-helix motif that is characteristic of the MarR family is an adaptation of the classical helix-turn-helix DNA-binding motif and in HucR corresponds to short $\alpha$-helices, $\alpha 4$ and $\alpha 5$, that pack at approximately $110^{\circ}$, followed by two anti-parallel $\beta$-strands ( $\beta 2$ and $\beta 3$ ) that form a $\beta$-hairpin referred to as the wing. Helix $\alpha 3$ appears to serve as a scaffold-like structure for this domain, as it mediates the majority of interactions within its own domain and those with the dimerization domain as well. The crystal structure reveals that the DNA-binding domains of HucR are completely independent of one another, in contrast to what is observed for MarR in which a salt-bridge links the two domains. ${ }^{8}$

The DNA-binding domains are positioned with a spacing between recognition helices that is compatible with DNA binding. HucR protects a $21 \mathrm{bp}$ site that features a pseudopalindromic sequence, and its recognition helices are predicted to engage consecutive DNA major grooves that lie on the same face of the DNA helix; ${ }^{3}$ OhrR, likewise, makes contacts across a 22 bp site containing a palindromic sequence, with recognition helices contacting consecutive DNA major grooves. ${ }^{10}$ Superposition of the HucR dimer onto the DNA-bound OhrR dimer structure (rmsd $1.9 \AA$ for $228 \mathrm{C}_{\alpha}$ atoms) suggests that no major reorientation of the domain interface is necessary to position the DNA-binding domains so that the recognition helices make contacts in the major groove of the hucR operator (Figure 2). This is true for the HucR dimer structures determined in an alternate crystal form as well; at room temperature, 

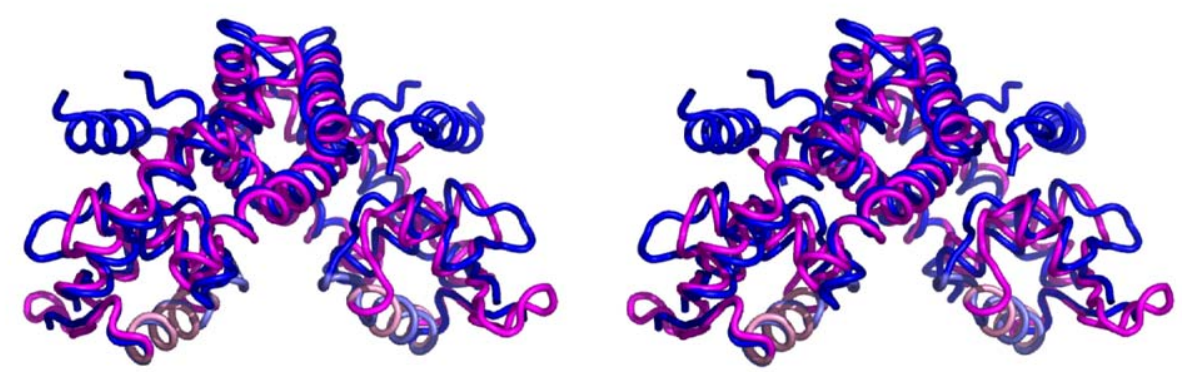

Figure 2. Superposition of HucR and OhrR structures. The DNA-bound structure of OhrR (1Z9C) is in magenta and HucR is in blue. The recognition helices are in lighter shades.

HucR crystallizes in space group $P 3_{1} 21$ with three dimers in the asymmetric unit. Although these crystals diffract to a lower resolution $(2.9 \AA)$, it is clear that all four HucR dimers (three in the $P 3_{1} 21$ crystal structure and one from the $P 6_{1}$ structure) have the same conformation at the dimer interface. This lack of conformational heterogeneity is in contrast to what was observed for ligand-free MexR, the structure of which revealed four independent dimers with significant conformational differences at the dimer interface. However, a localized conformational change in loop 99-104 in HucR is necessary to avoid steric clashes with the DNA. Thus, in contrast to what is observed for OhrR ${ }^{10}$ and $\mathrm{MexR}^{9}$ in the absence of DNA and ligand, the dimer interface of HucR under equivalent conditions is preconfigured, in terms of the spacing between the DNA-binding domains, for binding to DNA. That no major conformational changes of the HucR DNA-binding domains appear to be required for DNA binding may also explain the greater than tenfold higher affinity of HucR for its operator site compared to that of OhrR; ${ }^{3,18}$ HucR binds its cognate site with subnanomolar affinity even at concentrations of $\mathrm{NaCl}$ up to $300 \mathrm{mM}$ and in the presence of nanomolar concentrations of plasmid pGEM5 (data not shown). ${ }^{3}$ As described above, HucR contains an amino-terminal helix not found in other members of the MarR family; this helix is positioned at the amino-terminal end of the dimerization helix and appears to provide structural support for the protein-protein interface. Note in Figure 3 that Trp20 from $\alpha 1$ abuts dimerization helix $\alpha 2$. A conformation of DNA-binding domains that does not require extensive conformational changes to accommodate the DNA helix likely contributes to the high-affinity binding of HucR; such an affinity may be required to ensure effective repression of uricase expression.

The 17 residue $\beta$-hairpin wings of HucR jut out from the globular part of the DNA-binding domains and provide an exceptionally electropositive surface (Figure 1(c)): six of the 17 amino acid residues are arginine $(118,119,123,125,126$, and 130). Previous biochemical evidence has shown that HucR:R118A binds DNA with sixfold lower affinity relative to that of wild-type HucR. ${ }^{4}$ Moreover, the R118A mutant had no effect on sequence specificity of DNA binding, thus it is tempting to speculate that the wing is important (a)

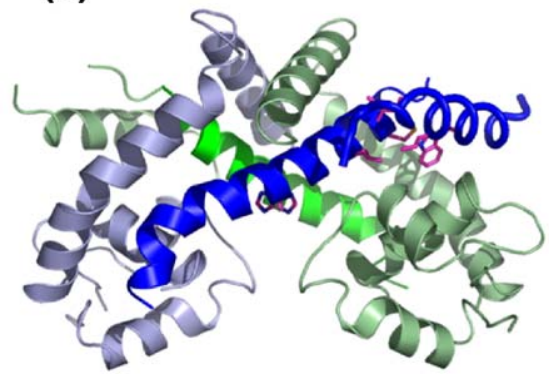

(b)

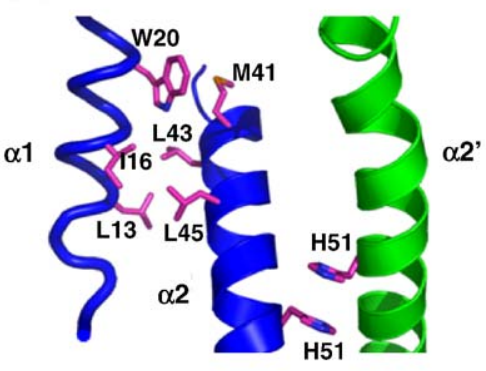

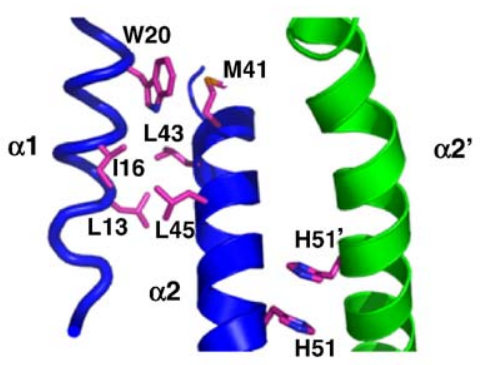

Figure 3. The packing interaction between $\alpha 1$ and $\alpha 2$ in HucR. (a) The HucR dimer with the dimerization helices colored in darker shades of blue and green. The side-chains of select amino acids are shown in atomic coloring with carbon in magenta. (b) A stereo detailed view of the interaction of $\alpha 1$ with helix $\alpha 2$, the HucR-specific and dimerization helices, respectively. 
for maintaining non-specific HucR/DNA interactions, similar to what is observed in other canonical winged helix proteins. ${ }^{22}$

Most winged helix proteins make sequencespecific contacts to their respective DNA targets via the recognition helix that immediately precedes the wing motif. ${ }^{22}$ In HucR, helix $\alpha 5$ (residues 101113) corresponds to the recognition helix, and is unusual, in that its amino terminus is a very short three residue $3_{10}$ helix that becomes a typical eight residue right-handed $\alpha$-helix. The electrostatic potential map shows that the HucR recognition helix is electropositive on both sides, a common feature for many winged-helix DNA-binding proteins. The recognition helix is anchored to the central scaffold helix of the DNA-binding domain $(\alpha 3)$ via a saltbridge between Arg106 and Asp73 and side-chain hydrogen bonds to Arg109 and Asn68. In addition, Lys113 makes a hydrogen bond to the main-chain carbonyl group of Gly66, located on a turn between helices $\alpha 2$ and $\alpha 3$. Furthermore, this Lys113/Gly66 interaction is functionally identical with the sidechain/main-chain H bonds in MarR (Lys81/Asp37) and MexR (Arg78/Asp34) and OhrR (Gln81/ Asn37). Such an interaction may be necessary to anchor the $C$ terminus of the recognition helix in a position suitable for DNA binding.

A structure-based sequence alignment of HucR, MarR, MexR, and OhrR is presented in Figure 4. Hydrophobic residues conserved throughout the DNA-binding domain appear to be important in stabilization of its overall fold. In fact, the DNAbinding domain has about twice as many invariant and/or highly conserved residues compared to the dimerization domain (Figure 4), while it represents just one-half of the common core of the MarR family. The bulk of the conserved amino acids are located in the hydrophobic core and include Leu67, Leu74, Leu75, Leu78, Leu87, Leu92, Leu110, Leu115, and Leu 132, and Ile107, Ile116, and Ile130. Another invariant residue from the MarR family of transcriptional regulators is Thr133, which is located at the proximal end of the wing between $\beta 3$ and helix $\alpha 6$. This residue appears to clamp the wing in place by making two hydrogen bonding interactions: its side-chain hydroxyl group makes an $\mathrm{H}$ bond with the backbone carbonyl group of Gly114 and the main chain nitrogen atom participates in an $\mathrm{H}$ bond with the backbone carbonyl group of Leu115. The length of the wing is further stabilized by typical $\beta$-sheet interactions and an additional salt-bridge between Arg131 and Glu117. There is insufficient electron density in the map to model the residues that comprise the distal wing (residues 121-127), most likely as a consequence of a high degree of flexibility in this region. Such mobility is common in winged-helix proteins, especially when the wing is not in a complex with its target DNA. 9,23

\section{The dimerization domain}

Helices $\alpha 2, \alpha 6, \alpha 7, \alpha 2^{\prime}, \alpha 6^{\prime}$, and $\alpha 7^{\prime}$ (where the prime denotes the other subunit) form the intertwined helical bundle that constitutes the dimerization domain. Helices $\alpha 2$ and $\alpha 2^{\prime}$ are oriented in an anti-parallel fashion and form the scaffold of the dimerization interface, while helices $\alpha 6$ and $\alpha 7$

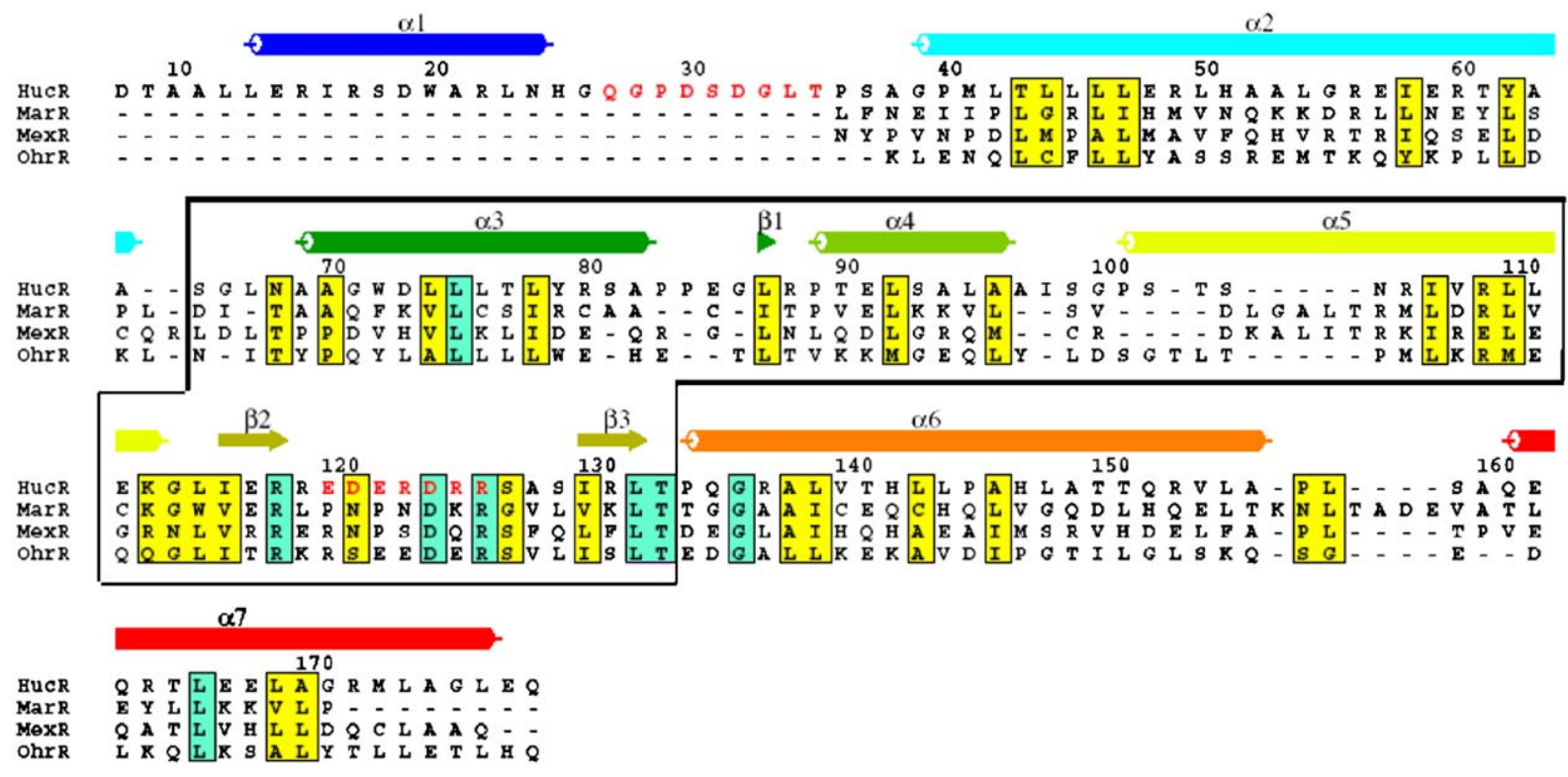

Figure 4. A structure-based sequence alignment of HucR, MexR, MarR and OhrR. The sequences of HucR, MexR, MarR and OhrR are aligned according to structural superpositions. The secondary structural elements of HucR are indicated and colored as in Figure 1(b). Residue numbering is according to HucR. Residues shown in red have no interpretable electron density in the structure of HucR and are not used in the superpositioning of the structures. Amino acids that are invariant (cyan) and similar (yellow) are indicated. The DNA-binding domain is encased by the black box. 
straddle helix $\alpha 2^{\prime}$, and helices $\alpha 6^{\prime}$ and $\alpha 7^{\prime}$ straddle $\alpha 2$. Helix $\alpha 1$, unique to the HucR structure, braces the amino-terminal end of $\alpha 2$ (Figure 3). As a consequence of the interdigitation of helices $\alpha 2, \alpha 6$, $\alpha 7$ and their dimer mates, a total of approximately $6300 \AA^{2}$ of surface area is buried at the dimerization interface. At the center of the 2-fold axis of the dimer is a $\pi$-stacking interaction between the imidazole rings of His51 and His51': the rings are parallel and separated by a distance of $3.6 \AA$ (Figures 3 and 5). We note that this stacking interaction would be disfavored upon protonation of both imidazole rings; the significance of the $\pi$-stacking interaction at a pivotal point in the dimer interface is discussed below. Helices $\alpha 2$ and $\alpha 2^{\prime}$ also interact through hydrophobic interactions that include Leu54/Leu44' and Leu50/Leu47', and there are two well-ordered pockets of water molecules located on either side of the stacked His51/His51' rings (Figure 5). The Cterminal helix $\alpha 7$ forms a coiled-coil leucine zipperlike structure with extensive hydrophobic interactions with $\alpha 2^{\prime}$, while $\alpha 6$ and $\alpha 2^{\prime}$ make only limited hydrophobic contacts. Additional interactions that contribute to dimer stabilization are the intermolecular salt-bridges Arg172/Glu162' $\left(\alpha 7 / \alpha 7^{\prime}\right)$ and Arg17/Glu167' ( $\left.\alpha 1 / \alpha 7^{\prime}\right)$. An intramolecular saltbridge between Arg153 and Glu57 stabilizes an interaction between the amino and carboxyl terminal helices that flank the DNA-binding domain.

The crystal structure of HucR and the structurebased alignment (Figure 4) reveal two conserved residues that might play a critical role in dimerization for members of the MarR family. Residues Leu166, which is strictly conserved in the MarR superfamily, and Leu166' of HucR make contact at the 2-fold rotation axis. They, along with other hydrophobic residues, collectively participate in the formation of the compact core of the dimerization domain, thus stabilizing helices $\alpha 7, \alpha 7^{\prime}, \alpha 2$, and $\alpha 2^{\prime}$ at this site. The MarR, MexR and OhrR equivalents (Leu139, Leu131 and Leu132, respectively) of Leu166 in those structures participate in similar van der Waals interactions. Leu158 from HucR, also conserved in MexR and MarR, serves to anchor a turn region located between helices $\alpha 6$ and $\alpha 7$ to the carboxyl-terminal helix $\alpha 7^{\prime}$ of the opposing monomer. Residues corresponding to Leu158 in both
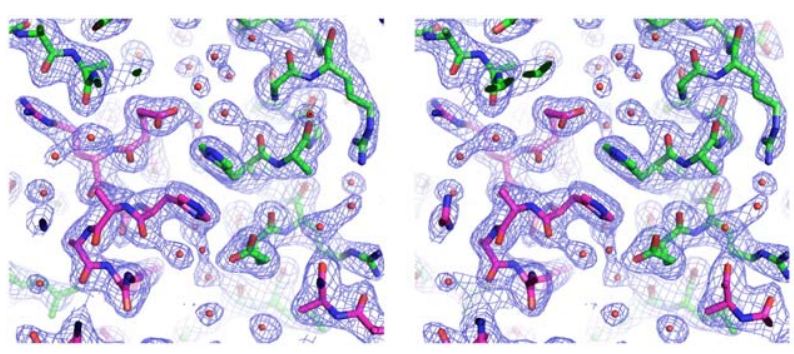

Figure 5. A region of the $2 F_{\mathrm{o}}-F_{\mathrm{c}}$ electron density map. The map is contoured at $1 \sigma$. The region corresponds to the dimer interface and the stacking of symmetry-related His51 residues is apparent.

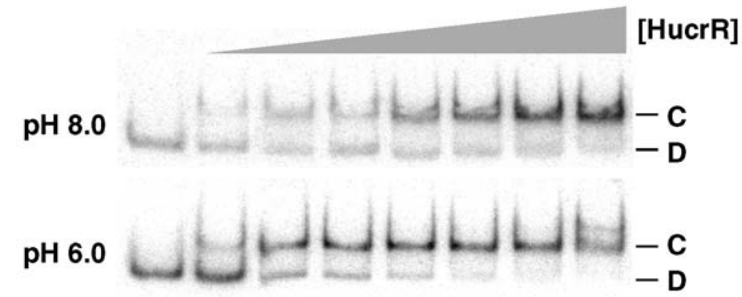

pH 5.0

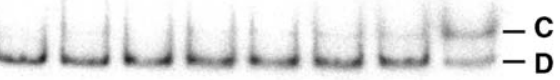

Figure 6. HucR-DNA complex formation is $\mathrm{pH}-$ dependent. EMSA showing the HucR operator DNA titrated with HucR at pH 8.0 (top panel), pH 6.0 (middle panel), and $\mathrm{pH} 5.0$ (bottom panel). Reactions at $\mathrm{pH} 8.0$ were electrophoresed at the same $\mathrm{pH}$, while reactions performed at $\mathrm{pH} 6.0$ and $\mathrm{pH} 5.0$ were loaded onto gels containing ME buffer (pH 6.0). Reactions in lane 1 contain DNA only. Reactions in lanes 2-8 contain identical concentrations of HucR $(0.1-20 \mathrm{nM})$. Complete saturation of reactions performed at $\mathrm{pH} 5.0$ is seen at higher [HucR]. Complex (C) and free DNA (D) are identified at the right.

MarR and MexR (Leu127 and Leu123, respectively) perform a similar structural role in those proteins and are likely to have a similar function in other MarR family members, given that this residue is highly conserved throughout the superfamily.

\section{The in vitro affinity of HucR for its operator is $\mathrm{pH}$-sensitive}

As described above, near the midpoint of the $40 \AA$ long $\alpha 2 / \alpha 2^{\prime}$ helices that provide the framework for the dimerization domain, the imidazole rings of His51 and His51' are stacked and separated by a distance of $3.6 \AA$ (Figures 3 and 5). At pH 7.0, the $\mathrm{pH}$ at which the crystals were obtained, the imidazole moieties would be expected to be deprotonated and, therefore, such an interaction is permissible. However, this configuration of His side-chains suggests that the dimer interface can serve as a $\mathrm{pH}$-sensor, and that a transient increase in proton concentration could lead to a conformational change induced by the repulsion of like charges. To assess the possibility that DNA binding may be compromised at lower $\mathrm{pH}$, we compared HucR-DNA complex formation at $\mathrm{pH} 8.0, \mathrm{pH} 6.0$ and $\mathrm{pH} 5.0$ (Figure 6). The DNA that contains the identified HucR-binding site extends from 180 bp upstream of the HucR translational start site to $61 \mathrm{bp}$ within the coding region. ${ }^{3}$ While no attenuation of DNA binding is seen upon a decrease of $\mathrm{pH}$ from 8.0 to $6.0\left(K_{\mathrm{d}}=0.20( \pm 0.01) \mathrm{nM}\right)$ to $6.0\left(K_{\mathrm{d}}=0.26( \pm 0.04) \mathrm{nM}\right)$, a further decrease in $\mathrm{pH}$ results in significant loss of complex formation, as reflected in an approximately tenfold higher $K_{\mathrm{d}}$. These observations are consistent with a $\mathrm{pH}$-dependent reorientation of DNA-binding domains that leads to compromised complex formation. As protonation of both histidine residues in the stacked 
pair is highly unfavorable, a significantly lowered histidine $\mathrm{pK}$ is expected, thus the impact on HucR binding to DNA is observed at a $\mathrm{pH}$ of 5 .

Structural studies of MarR family members indicate that conformational changes at the dimer interface result in repositioning of the DNA-binding domains. Thus, DNA binding is abrogated if the domains are repositioned such that the spacing between the recognition helices is not compatible with the distance between the major grooves of the cognate operator. In the MexR structure, determined in the absence of ligand or DNA, four independent dimers were observed and the relative orientations of the monomers within each dimer differ as a result of deviations at the dimerization interfaces, and structural heterogeneity in the monomer itself. ${ }^{9}$ The structure of the reduced form of the oxidative stress sensor OhrR in the absence and in the presence of DNA similarly revealed differences at the dimer interface. Furthermore, the authors propose that that oxidation of the conserved Cys, located on the OhrR $\alpha$-helix that corresponds to $\alpha 2$ in HucR, induces a conformational change at the dimer interface and thus abrogates DNA binding..$^{10}$ Our suggestion that proton-induced conformational changes at the HucR dimer interface attenuate DNA-binding affinity of the repressor is consistent with the flexibility at these interfaces observed in other MarR family members. It is not apparent from the data whether the conformational change induced by protonation is equivalent to that produced by the ligand uric acid. However, our observation that crystals transferred to mother liquor solution containing uric acid immediately crack is compatible with a significant ligand-induced conformational change.

A pair of similarly stacked histidine residues at a dimer interface has been shown to impart $\mathrm{pH}$ sensitivity to the dimerization of dynein light chain $\mathrm{LC} 8{ }^{24}$ but the physiological relevance of that $\mathrm{pH}-$ sensitive interface is not known. We note that the pH-sensitivity of HucR-DNA interaction is manifest at a $\mathrm{pH}$ at which uric acid would be protonated and thus even less soluble ( $\mathrm{pK} \sim 5.8$ ). It is tempting to speculate that a physiological role of a derepression of the uricase gene caused by a transient drop in cellular $\mathrm{pH}$ is the need to promote the conversion of uric acid to allantoin $\left(\mathrm{pK}_{\mathrm{a}} \sim 9.0\right)$, a compound with higher solubility.

\section{Materials and Methods}

\section{Protein expression and purification}

HucR was expressed in E. coli BL21(DE3)pLysS and purified with modifications to previously reported protocols. ${ }^{3}$ An overnight culture in Terrific Broth containing $100 \mu \mathrm{g} / \mathrm{ml}$ of ampicillin and $34 \mu \mathrm{g} / \mathrm{ml}$ of chloramphenicol was diluted $1: 100(\mathrm{v} / \mathrm{v})$ and incubated at $37^{\circ} \mathrm{C}$. HucR expression was induced at $A_{600}=0.6$ with $0.1 \mathrm{mM}$ isopropyl- $\beta$-D-thiogalactopyranoside (IPTG) and the temperature was reduced to $20^{\circ} \mathrm{C}$. After $24 \mathrm{~h}$ the cells were pelleted and frozen at $-80{ }^{\circ} \mathrm{C}$. Cells were lysed with Bugbuster (Novagen) and sonicated in the presence of DNase I and the protease inhibitors pepstatin and leupeptin. The clarified lysate was applied to a $10 \mathrm{ml}$ Affi-Gel Blue Gel (Biorad) column equilibrated with buffer A (10 mM Hepes ( $\mathrm{pH} 7.0), 50 \mathrm{mM} \mathrm{KCl}, 2 \mathrm{mM} \beta-$ mercaptoethanol). HucR was eluted batch-wise with buffer A supplemented with $1 \mathrm{M} \mathrm{KCl}$. HucR binds neither DEAE-cellulose nor CM-cellulose equilibrated in buffer A, thus passage through these two columns removed contaminating proteins. Subsequently, the protein was applied to a hydroxylapatite column equilibrated with buffer $\mathrm{A}$ and eluted with $1 \mathrm{M}$ potassium phosphate in buffer A. Finally, HucR was applied to a Superdex-200 size-exclusion column. Protein purity was assessed by SDS-PAGE. HucR was concentrated to $15 \mathrm{mg} / \mathrm{ml}$ and flash-frozen in liquid nitrogen for storage at $-80^{\circ} \mathrm{C}$.

Selenomethionine (SeMet)-labeled HucR was expressed using E. coli BL21(DE3)pLysS cells. An overnight culture grown in LB was used to make a 1:100 (v/v) dilution with fresh M9 medium supplemented with $2 \mathrm{mM} \mathrm{MgSO}_{4}$, $0.1 \mathrm{mM} \mathrm{CaCl}$, and $4 \mathrm{~g} / 1$ of glycerol. Cells were grown until $A_{600}$ reached 0.5 , at which point $100 \mathrm{mg} / 1$ of amino acids threonine, lysine, phenylalanine, and $50 \mathrm{mg} / 1$ of

Table 1. Summary of data collection

\begin{tabular}{|c|c|c|c|c|c|}
\hline & \multicolumn{3}{|c|}{ SeMet MAD } & \multicolumn{2}{|c|}{ Native } \\
\hline & Peak & Inflection & Remote & Native 1 & Native 2 \\
\hline Space group & $P 6_{1} 22$ & & & $P 6_{1}$ & $P 3_{1} 21$ \\
\hline Wavelength $(\AA)$ & 0.9795 & 0.9798 & 0.9500 & 1.380 & 0.9505 \\
\hline \multicolumn{6}{|l|}{ Unit cell parameters } \\
\hline$a(\AA)$ & 44.9 & & & 45.0 & 77.3 \\
\hline$b(\AA)$ & 44.9 & & & 45.0 & 77.3 \\
\hline$c(\AA)$ & 286.4 & & & 284.6 & 266.4 \\
\hline$\alpha$ (deg.) & 90 & & & 90 & 90 \\
\hline$\beta$ (deg.) & 90 & & & 90 & 90 \\
\hline$\gamma($ deg.) & 120 & & & 120 & 120 \\
\hline Resolution range $^{\mathrm{a}}(\boldsymbol{\AA})$ & $50-2.65(2.74)$ & $50-2.65(2.74)$ & $50-2.65(2.74)$ & $50-2.30(2.38)$ & $50-2.90(3.00)$ \\
\hline Unique reflections & 5659 & 5714 & 5544 & 14,303 & 21,171 \\
\hline Completeness $^{\mathrm{a}}(\%)$ & $98.9(99.8)$ & $98.9(100)$ & $96.4(99.6)$ & $98.8(95.8)$ & $98.6(99.7)$ \\
\hline Redundancy ${ }^{\mathrm{a}}$ & $13.1(9.2)$ & $10.0(9.3)$ & $5.8(7.7)$ & $4.4(3.8)$ & $3.5(3.5)$ \\
\hline$I / \sigma^{\mathrm{a}}$ & $13.9(4.2)$ & $15.9(5.0)$ & $9.3(4.0)$ & $12.1(8.1)$ & $8.9(2.7)$ \\
\hline$R_{\text {sym }}{ }^{\mathrm{b}}(\%)$ & $13.3(49.0)$ & $11.0(47.3)$ & $12.4(47.6)$ & $7.5(20.4)$ & $12.6(45.5)$ \\
\hline
\end{tabular}


leucine, valine, and isoleucine were added to the medium to inhibit the methionine biosynthetic pathway. Cells were grown for an additional $45 \mathrm{~min}$ to deplete residual cellular levels of L-methionine, then $0.2 \mathrm{mM}$ IPTG and $50 \mathrm{mg} / 1$ of SeMet were added, and the growth temperature was reduced to $24{ }^{\circ} \mathrm{C}$. Production of SeMet-labeled HucR was allowed to proceed for $24 \mathrm{~h}$ before the cells were harvested. Purification of SeMet HucR was carried out as described above for the native HucR protein, with the exception that buffer A was supplemented with $10 \mathrm{mM} \beta$-mercaptoethanol.

\section{Crystallization and data collection}

HucR crystals were grown by the hanging-drop, vapordiffusion method with 1:1 (v/v) mixtures of $12 \mathrm{mg} / \mathrm{ml}$ of protein and 22\% (w/v) PEG 3350, $500 \mathrm{mM} \mathrm{MgCl}_{2}, 100 \mathrm{mM}$ Bis-Tris (pH 7.0 at $4{ }^{\circ} \mathrm{C}$ ). Long, rod-like hexagonal crystals appeared after four days for the native HucR and after two weeks for the SeMet-labeled HucR. Native and SeMet crystals belong to the hexagonal space groups $P 6_{1}$ $(a=b=45.0, c=284.6)$ and P6 $22 \quad(a=b=44.9, c=286.4)$, and were found to contain two molecules and one molecule within their asymmetric unit, respectively. A third crystal form of space group $P 3_{1} 21 \quad(a=b=77.3$, $c=266.4$ ) was produced at room temperature in the same conditions supplemented with $1 \mathrm{mM} \mathrm{Pb}\left(\mathrm{NO}_{3}\right)_{2}$. The room temperature crystal form, which has three dimers in the asymmetric unit, was merohedrally twinned with a twin fraction of 0.266 as determined in CNS. ${ }^{25}$

Crystals were flash-frozen in liquid nitrogen and diffraction data were collected with a MarCCD detector $(165 \mathrm{~mm})$ at the protein crystallography beamline of the Center for Advanced Microstructures and Devices (CAMD, Louisiana State University). All data processing and scaling was carried out with HKL2000 software, ${ }^{26}$ with default $\sigma$ cutoffs of -3.0 applied. Data were collected at three wavelengths for the SeMet HucR crystals to $2.65 \AA$ resolution. All data collection statistics are given in Table 1.

\section{Structure determination and refinement}

Initial phases (Table 2) were obtained by MAD phasing to $2.8 \AA$ resolution with the SeMet data sets. Two of a possible four SeMet residues were apparent in a threewavelength anomalous difference Patterson calculated with CNS. After density modification, which resulted in an overall figure of merit of 0.49 , a primarily polyalanine model was built with the program O. ${ }^{27}$ Phase combination of model and experimental phases was performed to improve map quality until approximately two-thirds of the model was built. At this point $2 F_{\mathrm{o}}-F_{\mathrm{c}}$ maps at $2.65 \AA$ resolution were calculated for model building. Composite omit maps were utilized throughout the model-building process to avoid register errors.

Once an $R_{\text {free }}$ value of 0.43 was obtained, the model derived from the SeMet data was positioned in the wildtype $P 6_{1}$ unit cell for further refinement with noncrystallographic symmetry (NCS) weights of 400 . Water molecules were added at positions of $3 \sigma$ peaks in the electron density map located within hydrogen bonding distance of a donor or acceptor atom. Two peaks that appeared to be larger than those assigned to water were refined as chloride ions, due to an absolute requirement for a high concentration of $\mathrm{MgCl}_{2}$ in the crystallization conditions and the presence of a positive charge in proximity to the peak. The final $2.3 \AA$ resolution model $\left(R_{\text {cyst }}=23.5 \% R_{\text {free }}=29.0 \%\right.$, no $\sigma$ cutoff $)$ includes 157 of a
Table 2. MAD phasing and structure refinement statistics

\begin{tabular}{|c|c|c|c|}
\hline & $\begin{array}{l}\text { SeMet } \\
P 6_{1} 22\end{array}$ & $\begin{array}{c}\text { Native } \\
P 6_{1} \\
\end{array}$ & $\begin{array}{r}\text { Native } \\
P 3_{1} 21 \\
\end{array}$ \\
\hline \multicolumn{4}{|l|}{ A. Phasing } \\
\hline Resolution range $(\AA)$ & $500-2.8$ & & \\
\hline \multicolumn{4}{|l|}{ Figure of merit } \\
\hline Centrics & 0.68 & & \\
\hline Acentrics & 0.44 & & \\
\hline All & 0.49 & & \\
\hline \multicolumn{4}{|l|}{ Phasing power } \\
\hline Centrics & 1.55 & & \\
\hline Acentrics & 1.41 & & \\
\hline All & 1.45 & & \\
\hline \multicolumn{4}{|l|}{ B. Refinement } \\
\hline Resolution range $(\AA)$ & & $500-2.3$ & $50-2.9$ \\
\hline$R_{\text {cryst }} \mathrm{b}^{(\%)}$ & & 23.5 & 22.1 \\
\hline$R_{\text {free }}{ }^{\mathrm{c}}(\%)$ & & 29.0 & 30.4 \\
\hline \multicolumn{4}{|l|}{ No. non-hydrogen atoms } \\
\hline Protein & & 2358 & 7088 \\
\hline Water & & 187 & 88 \\
\hline Chloride & & 2 & na \\
\hline \multicolumn{4}{|l|}{ rmsd from ideal geometry } \\
\hline Bond lengths $(\AA)$ & & 0.006 & 0.007 \\
\hline Bond angles (deg.) & & 1.20 & 1.30 \\
\hline \multicolumn{4}{|l|}{ Average $B$-factor } \\
\hline Protein $\left(\AA^{2}\right)$ & & 52 & 27.3 \\
\hline Water $\left(\AA^{2}\right)$ & & 51 & 19.2 \\
\hline Chloride $\left(\AA^{2}\right)$ & & 41 & na \\
\hline \multicolumn{4}{|l|}{ Ramachandran plot } \\
\hline Most favored regions (\%) & & 90.9 & 87.0 \\
\hline $\begin{array}{l}\text { Additionally allowed } \\
\text { regions }(\%)\end{array}$ & & 8.3 & 10.3 \\
\hline $\begin{array}{l}\text { Generously allowed } \\
\text { regions }(\%)\end{array}$ & & 0.8 & 1.4 \\
\hline Disallowed region (\%) & & 0.0 & 1.4 \\
\hline
\end{tabular}

${ }^{a}$ Merohedrally twinned dataset containing a twin fraction of 0.266 as determined by CNS.

b $R=\sum\left\|F_{\mathrm{o}}|-| F_{\mathrm{c}}\right\| / \sum\left|F_{\mathrm{o}}\right|$, where $F_{\mathrm{o}}$ and $F_{\mathrm{c}}$ are the observed and calculated structure factor amplitudes.

${ }^{c} R_{\text {free }}$ was calculated by withholding $10 \%$ of the total reflections from refinement.

total of 181 residues: The amino terminus (residues 1-7), the last two residues on the carboxyl terminus, the wing residues 121-127, and loop residues 27-35 were not visible in the electron density map. In addition, amino acid residues 25, 36, 88, and 120 have no convincing electron density for side-chain placement. The significant portion of unobserved and presumably disordered polypeptide $(13 \%)$ likely limits further refinement, and the value achieved for $R_{\text {free }}$ is similar to what has been reported for other MarR family members. Refinement statistics are shown in Table 2 and a representative $2 F_{\mathrm{o}}-$ $F_{c}$ electron density map contoured at $1 \sigma$ is depicted in Figure 5. All Figures were prepared using PYMOL $\dagger$, $\mathrm{GRASP}^{28}$ or $\mathrm{O} .^{27}$

The dimer was positioned in the $P 3_{1} 21$ unit cell by molecular replacement as implemented in CNS. The structure was refined to a resolution of $2.9 \AA$ to give a final $R_{\text {cryst }}=22.2 \%$ and $R_{\text {free }}=30.5 \%$. The six dimers in the asymmetric unit were restrained by NCS weights of 500 .

\section{Electrophoretic mobility-shift assay}

For electrophoretic mobility-shift assays (EMSA), $0.1 \mathrm{nM}{ }^{32}$ P-labeled $241 \mathrm{bp}$ DNA containing the HucR-

$\dagger$ http:/ / www.pymol.org 
binding site ${ }^{3}$ was titrated with HucR in a total reaction volume of $10 \mu \mathrm{l}$. Reaction conditions included the identified buffer $(40 \mathrm{mM}$ Tris- $\mathrm{HCl}(\mathrm{pH} 8.0)$ or $40 \mathrm{mM}$ Mes (pH 6.0 or pH 5.0) and 0.1 mM EDTA, $0.075 \%(w / v)$ Brij58, $200 \mathrm{mM} \mathrm{NaCl}, 50 \mu \mathrm{g} / \mathrm{ml}$ of bovine serum albumin and $4 \%(\mathrm{v} / \mathrm{v})$ glycerol. Reactions were incubated at $22{ }^{\circ} \mathrm{C}$ for $30 \mathrm{~min}$ before loading onto $7 \%(\mathrm{w} / \mathrm{v})$ polyacrylamide gels (39:1 (w/w) acrylamide:bisacrylamide) in TE buffer (40 mM Tris, 1 mM EDTA, pH 8.0) or ME buffer (50 mM Mes, 1 mM EDTA, pH 6.0 or $\mathrm{pH}$ 5.0). Gels were run for $30 \mathrm{~min}$ at $20 \mathrm{~mA}$ at room temperature before loading the samples with the power on. After electrophoresis, gels were dried and protein-DNA complexes were visualized and quantified by phosphorimaging, using software supplied by the manufacturer (ImageQuant 1.1). Binding constants were calculated as described, ${ }^{3}$ and are reported in terms of [HucR dimer]. All experiments were performed at least in triplicate.

\section{Data bank accession code}

Coordinates and structure factors have been deposited in the RCSB Protein Data Bank with accession code 2FBK.

\section{Acknowledgements}

We thank Henry Bellamy and David Neau at the GCPCC beamline of the Center for Advanced Microstructures and Devices for their assistance with MAD data collection. We thank S. Pakhomova, P. Bilder, and M. Oldham for providing valuable insight throughout this project. This work was supported, in part, by the Louisiana Governors Biotechnology Initiative (MEN) and by the LSU AgCenter (to T.B.).

\section{References}

1. Makarova, K. S., Aravind, L., Wolf, Y. I., Tatusov, R. L., Minton, K. W., Koonin, E. V. \& Daly, M. J. (2001). Genome of the extremely radiation-resistant bacterium Deinococcus radiodurans viewed from the perspective of comparative genomics. Microbiol. Mol. Biol. Rev. 65, 44-79.

2. White, O., Eisen, J. A., Heidelberg, J. F., Hickey, E. K., Peterson, J. D., Dodson, R. J. et al. (1999). Genome sequence of the radioresistant bacterium Deinococcus radiodurans $\mathrm{R} 1$. Science, 286, 1571-1577.

3. Wilkinson, S. P. \& Grove, A. (2004). HucR, a novel uric acid-responsive member of the MarR family of transcriptional regulators from Deinococcus radiodurans. J. Biol. Chem. 279, 51442-51450.

4. Wilkinson, S. P. \& Grove, A. (2005). Negative cooperativity of uric acid binding to the transcriptional regulator HucR from Deinococcus radiodurans. J. Mol. Biol. 350, 617-630.

5. Hooper, D. C., Spitsin, S., Kean, R. B., Champion, J. M., Dickson, G. M., Chaudhry, I. \& Koprowski, H. (1998). Uric acid, a natural scavenger of peroxynitrite, in experimental allergic encephalomyelitis and multiple sclerosis. Proc. Natl Acad. Sci. USA, 95, 675-680.

6. Kean, R. B., Spitsin, S. V., Mikheeva, T., Scott, G. S. \& Hooper, D. C. (2000). The peroxynitrite scavenger uric acid prevents inflammatory cell invasion into the central nervous system in experimental allergic encephalomyelitis through maintenance of bloodcentral nervous system barrier integrity. J. Immunol. 165, 6511-6518.

7. Ames, B. N., Cathcart, R., Schwiers, E. \& Hochstein, P. (1981). Uric acid provides an antioxidant defense in humans against oxidant- and radical-caused aging and cancer: a hypothesis. Proc. Natl Acad. Sci. USA, 78, 6858-6862.

8. Alekshun, M. N., Levy, S. B., Mealy, T. R., Seaton, B. A. \& Head, J. F. (2001). The crystal structure of MarR, a regulator of multiple antibiotic resistance, at $2.3 \AA$ resolution. Nature Struct. Biol. 8, 710-714.

9. Lim, D., Poole, K. \& Strynadka, N. C. (2002). Crystal structure of the MexR repressor of the mexRAB-oprM multidrug efflux operon of Pseudomonas aeruginosa. J. Biol. Chem. 277, 29253-29259.

10. Hong, M., Fuangthong, M., Helmann, J. D. \& Brennan, R. G. (2005). Structure of an OhrR-ohrA operator complex reveals the DNA binding mechanism of the MarR family. Mol. Cell, 20, 131-141.

11. Egland, P. G. \& Harwood, C. S. (1999). BadR, a new MarR family member, regulates anaerobic benzoate degradation by Rhodopseudomonas palustris in concert with AadR, an Fnr family member. J. Bacteriol. 181, 2102-2109.

12. Bartels, F. W., Baumgarth, B., Anselmetti, D., Ros, R. \& Becker, A. (2003). Specific binding of the regulatory protein ExpG to promoter regions of the galactoglucan biosynthesis gene cluster of Sinorhizobium meliloti -a combined molecular biology and force spectroscopy investigation. J. Struct. Biol. 143, 145-152.

13. Alekshun, M. N. \& Levy, S. B. (1999). The mar regulon: multiple resistance to antibiotics and other toxic chemicals. Trends Microbiol. 7, 410-413.

14. Alekshun, M. N. \& Levy, S. B. (1999). Alteration of the repressor activity of MarR, the negative regulator of the Escherichia coli marRAB locus, by multiple chemicals in vitro. J. Bacteriol. 181, 4669-4672.

15. Li, X. Z. \& Poole, K. (1999). Organic solvent-tolerant mutants of Pseudomonas aeruginosa display multiple antibiotic resistance. Can. J. Microbiol. 45, 18-22.

16. Srikumar, R., Paul, C. J. \& Poole, K. (2000). Influence of mutations in the mexR repressor gene on expression of the MexA-MexB-oprM multidrug efflux system of Pseudomonas aeruginosa. J. Bacteriol. 182, 1410-1414.

17. Fuangthong, M. \& Helmann, J. D. (2002). The OhrR repressor senses organic hydroperoxides by reversible formation of a cysteine-sulfenic acid derivative. Proc. Natl Acad. Sci. USA, 99, 6690-6695.

18. Fuangthong, M., Atichartpongkul, S., Mongkolsuk, S. \& Helmann, J. D. (2001). OhrR is a repressor of ohrA, a key organic hydroperoxide resistance determinant in Bacillus subtilis. J. Bacteriol. 183, 4134-4141.

19. Wu, R. Y., Zhang, R. G., Zagnitko, O., Dementieva, I., Maltzev, N., Watson, J. D. et al. (2003). Crystal structure of Enterococcus faecalis SlyA-like transcriptional factor. J. Biol. Chem. 278, 20240-20244.

20. Evans, K., Adewoye, L. \& Poole, K. (2001). MexR repressor of the mexAB-oprM multidrug efflux operon of Pseudomonas aeruginosa: identification of MexR binding sites in the mexA-mexR intergenic region. J. Bacteriol. 183, 807-812.

21. Martin, R. G. \& Rosner, J. L. (1995). Binding of purified multiple antibiotic-resistance repressor protein (MarR) to mar operator sequences. Proc. Natl Acad. Sci. USA, 92, 5456-5460. 
22. Gajiwala, K. S. \& Burley, S. K. (2000). Winged helix proteins. Curr. Opin. Struct. Biol. 10, 110-116.

23. De Silva, R. S., Kovacikova, G., Lin, W., Taylor, R. K. Skorupski, K. \& Kull, F. J. (2005). Crystal structure of the virulence gene activator AphA from Vibrio cholerae reveals it is a novel member of the winged helix transcription factor superfamily. J. Biol. Chem. 280, 13779-13783.

24. Nyarko, A., Cochrun, L., Norwood, S., Pursifull, N., Voth, A. \& Barbar, E. (2005). Ionization of His 55 at the dimer interface of dynein light-chain LC8 is coupled to dimer dissociation. Biochemistry, 44, 14248-14255.

25. Brunger, A. T., Adams, P. D., Clore, G. M., DeLano,
W. L., Gros, P., Grosse-Kunstleve, R. W. et al. (1998). Crystallography \& NMR system: a new software suite for macromolecular structure determination. Acta Crystallog. sect. D, 54, 905-921.

26. Otwinoski, Z. \& Minor, M. (1997). Processing of X-ray diffraction data collected in oscillation mode. Methods Enzmol. 276, 307-326.

27. Jones, T. A., Zou, J. Y. \& Cowan, S. W. (1991). Kjeldgaard, Improved methods for building protein models in electron density maps and the location of errors in these models. Acta Crystallog. sect. A, 47, $110-119$.

28. Honig, B. \& Nicholls, A. (1995). Classical electrostatics in biology and chemistry. Science, 268, 1144-1149.

Edited by K. Morikawa

(Received 17 March 2006; received in revised form 28 April 2006; accepted 2 May 2006) Available online 19 May 2006 